TAIWANESE JOURNAL OF MATHEMATICS

Vol. 9, No. 4, pp. 639-660, December 2005

This paper is available online at http://www.math.nthu.edu.tw/tjm/

\title{
GROUP THEORETIC METHOD ANALYSIS FOR UNSTEADY BOUNDARY LAYER FLOW NEAR A STAGNATION POINT
}

\author{
I. A. Hassanien, A. A. Salama and H. A. Hosham
}

\begin{abstract}
An analysis of the problem of unsteady laminar boundary layer near a stagnation point is presented. The transformation group theoretic approach is applied to this problem. The application of two-parameter groups reduces the number of independent variables in the governing system consisting of partial differential equations and a set of auxiliary conditions from three to only one independent variable, and consequently the system of governing partial differential equations with boundary conditions reduces to a system of ordinary differential equations with appropriate boundary conditions. The possible forms of the main stream velocity and the surface temperature variations with position and time are derived. It is shown how not only we recover many of the previously known exact solutions but also find some completely new forms.
\end{abstract}

\section{INTRODUCTION}

Almost a century ago Prandtl realised the key part that boundary layers play in determining accurately the flow of certain fluids. He showed for slightly viscous flows that although viscosity is negligible in the bulk of the flow, it assumes a vital role near boundaries.

The quest for exact solutions of the boundary-layer equations has a long history. Blasius (1908) used a scaling to obtain the similarity solution of the steady boundary layer flow of a flat plate. Further work Burde' (1990, 1994, 1995, 1996) obtained similarity solution corresponding to stagnation point flows, flows past wedges, jets and flows near an oscillating plate. Jones and Watson (1963) have given a comprehensive account of many of the classical exact solutions of the boundary-layer equations including Falkner-Skan forms and the asymptotic suction profile. The two-

Received August 14, 2003; accepted March 12, 2004.

Communicated by Robert Finn.

2000 Mathematics Subject Classification: 70G65, 76D05.

Key words and phrases: Similar solution, Group theoretic method, Boundary layer, Stagnation point. 


\section{Nomenclature}

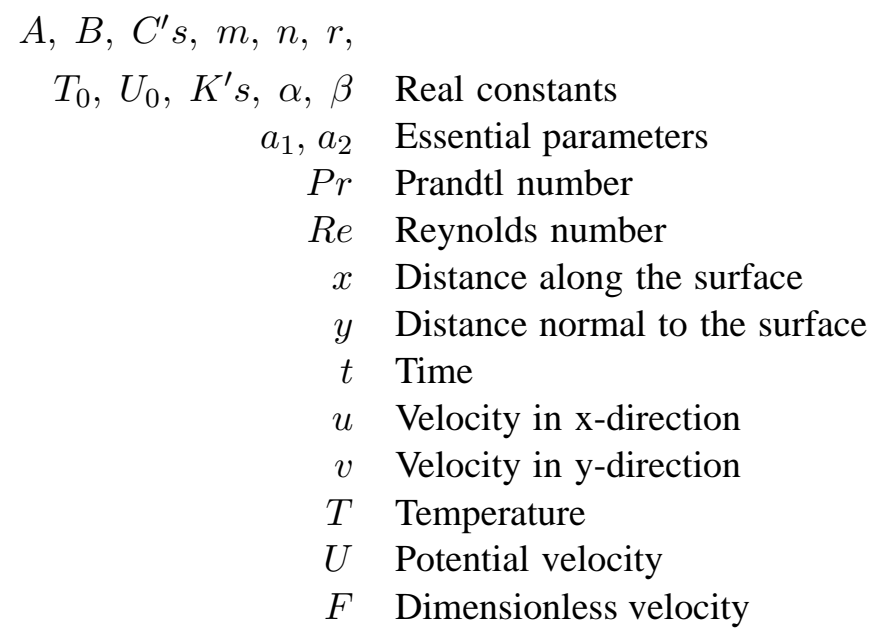

Greek symbols

$\eta \quad$ Dimensionless coordinate

$\theta$ Dimensionless temperature

$\psi \quad$ Stream function

Subscripts

$w \quad$ Surface conditions

$\infty$ Conditions far a way form the surface

parameter groups provide a powerful tool because they are not based on linear operators, superposition, or any other aspects of linear solution techniques. Ma and Hui (1990) used the method of Lie group transformations to derive all possible group-invariant similarity solutions to the problem of unsteady two-dimensional boundary-layer flow of an incompressible fluid. This method is based on nonlinear superposition is then used to generate further similarity solution which are not groupinvariant. Recently, the symmetry analysis applied to boundary layer equations is, introduced by Cantwell (2003).

Variants of the two-parameter groups have been applied to several problems within fluid mechanics. The new systematic formalism introduced here is well studied for the similarity analysis of unsteady laminar boundary layer in an incompressible stagnation point flow. This new systematic formalism reduces the number of independent variables in the system which consists, in general, of a set of partial differential equations and auxiliary conditions (such as boundary and/or initial conditions) by two. These reduce the system of partial differential equations to a system of ordinary differential equations with the appropriate boundary conditions. The general analysis is developed in this study to cases of the wall temperature $T_{w}$ and potential velocity $U$ in which they have several forms in the position and time. 
Throughout we make frequent contact with previously derived similarity solutions and show that while the two-parameter groups recover many of these forms they also find several completely new ones.

\section{Mathematical Analysis}

Consider an unsteady, two-dimensional, laminar, boundary layer flow near a stagnation point. We use Cartesian coordinate system oxyz, the governing equations can be approximated by the dimensionless in the form:

$$
\begin{gathered}
\frac{\partial u}{\partial x}+\frac{\partial v}{\partial y}=0 \\
\frac{\partial u}{\partial t}+u \frac{\partial u}{\partial x}+v \frac{\partial u}{\partial y}=\frac{\partial^{2} u}{\partial y^{2}}+\frac{\partial U}{\partial t}+U \frac{\partial U}{\partial x} \\
\frac{\partial T}{\partial t}+u \frac{\partial T}{\partial x}+v \frac{\partial T}{\partial y}=\frac{1}{\operatorname{Pr}} \frac{\partial^{2} T}{\partial y^{2}}
\end{gathered}
$$

with the boundary conditions

(4) $y=0: u=0, v=v_{w}=$ Const., $T=T_{w}(x, t), y \rightarrow \infty: u=U(x, t), T=T_{\infty}$,

where

$$
\begin{aligned}
& x=\frac{\bar{x}}{L}, y=\frac{\bar{y}}{L} \sqrt{R e}, t=\frac{U^{*}}{L} \bar{t}, u=\frac{\bar{u}}{U^{*}}, v=\frac{\bar{v}}{U^{*}} \sqrt{R} e, \\
& T-T_{\infty}=\frac{L}{U^{*^{2}}}\left(\bar{T}-\bar{T}_{\infty}\right), \theta=\frac{T-T_{\infty}}{T_{w}-T_{\infty}}=\frac{T-T_{\infty}}{T_{1}} .
\end{aligned}
$$

These equations are presented by Schlichting and Gersten [20]. If we introduce the non-dimensional stream function $\psi(x, y, t)$ such that $u=\frac{\partial \psi}{\partial y}, v=-\frac{\partial \psi}{\partial x}$ and the non-dimensional temperature given by (5), Eqs. (1-3) become:

$$
\begin{gathered}
\frac{\partial^{2} \psi}{\partial y \partial t}+\frac{\partial \psi}{\partial y} \frac{\partial^{2} \psi}{\partial y \partial x}-\frac{\partial \psi}{\partial x} \frac{\partial^{2} \psi}{\partial y^{2}}=\frac{\partial^{3} \psi}{\partial y^{3}}+\frac{\partial U}{\partial t}+U \frac{\partial U}{\partial x} \\
T_{1} \frac{\partial \theta}{\partial t}+\theta \frac{\partial T_{1}}{\partial t}+\frac{\partial \psi}{\partial y}\left(T_{1} \frac{\partial \theta}{\partial x}+\theta \frac{\partial T_{1}}{\partial x}\right)-T_{1} \frac{\partial \psi}{\partial x} \frac{\partial \theta}{\partial y}=\frac{1}{P r} T_{1} \frac{\partial^{2} \theta}{\partial y^{2}}
\end{gathered}
$$

with the boundary conditions

(8) $y=0: \frac{\partial \psi}{\partial y}=0, \frac{\partial \psi}{\partial x}=-v_{w}, \theta=1, y \rightarrow \infty: \frac{\partial \psi}{\partial y}=U(x, t), \theta=0$. 


\section{The Group of Transformations}

In this section, two-parameter transformation group is applied to the system of Eqs.(6-7) with the boundary conditions (8). The system of equations reduces to a system of ordinary differential equations in a single independent variable with the appropriate boundary conditions. The procedure is initiated with the group $G$, a class of two-parameter group of the form:

$$
G: \bar{S}=C^{S}\left(a_{1}, a_{2}\right) S+K^{S}\left(a_{1}, a_{2}\right),
$$

where $S$ stands for $x, y, t, \psi, T_{1}, U$ and $\theta$ and the $C^{S}$ and $K^{S}$ are realvalued at least differentiable in their arguments $\left(a_{1}, a_{2}\right)$, the parameters of the group. For more details about the transformation (9) see Moran and Gaggioli (1970), Ovsiannikov (1982) and Abd-el-Malek and El-Mansi (2000). Thus, in the notation of the given representation, the present analysis is initiated with a class $C_{G}$ of two-parameter transformation group in the form

$$
G:\left\{\begin{array}{l}
\bar{S}:\left\{\begin{array}{l}
\bar{x}=C^{x}\left(a_{1}, a_{2}\right) x+K^{x}\left(a_{1}, a_{2}\right) \\
\bar{y}=C^{y}\left(a_{1}, a_{2}\right) y+K^{y}\left(a_{1}, a_{2}\right) \\
\bar{t}=C^{t}\left(a_{1}, a_{2}\right) t+K^{t}\left(a_{1}, a_{2}\right)
\end{array}\right. \\
\bar{\psi}=C^{\psi}\left(a_{1}, a_{2}\right) \psi+K^{\psi}\left(a_{1}, a_{2}\right) \\
\bar{\theta}=C^{\theta}\left(a_{1}, a_{2}\right) \theta+K^{\theta}\left(a_{1}, a_{2}\right) \\
\bar{T}_{1}=C^{T_{1}}\left(a_{1}, a_{2}\right) T_{1}+K^{T_{1}}\left(a_{1}, a_{2}\right) \\
\bar{U}=C^{U}\left(a_{1}, a_{2}\right) U+K^{U}\left(a_{1}, a_{2}\right),
\end{array}\right.
$$

which possesses complete sets of absolute invariants $\eta(x, y, t)$ and $\chi_{i}\left(x, y, t, \psi, \theta, T_{1}\right), \quad i=$ $1,2,3$ where $\chi_{i}$ are the three absolute invariants corresponding to $\psi, \theta, U$ and $T_{1}$. If $\eta$ is the absolute invariant of the independent variables, then $\chi_{i}=F_{i}(\eta), \quad i=1,2,3$.

\section{The InVARiance AnALYsis}

The transformation for the derivatives of the differential equations (6-8), are obtained directly from $G$ via chain rule operations

(11) $\bar{S}_{\bar{x}}=\left(C^{S} / C^{x}\right) S_{x}, \bar{S}_{\bar{x} \bar{y}}=\left(C^{S} / C^{x} C^{y}\right) S_{x y}, \ldots, \bar{S}_{\bar{x} \bar{y} \bar{z}}=\left(C^{S} / C^{x} C^{y} C^{z}\right) S_{x y z}$.

Eq. (6) is said to be invariantly transformed under (9) and (11) whenever

$$
\begin{aligned}
& \bar{\Psi}_{\bar{y} \bar{t}}+\bar{\Psi}_{\bar{y}} \bar{\Psi}_{\bar{y} \bar{x}}-\bar{\Psi}_{\bar{x}} \bar{\Psi}_{\bar{y} \bar{y}}-\bar{\Psi}_{\bar{y} \bar{y} \bar{y}}-\bar{U}_{\bar{t}}-\bar{U} \bar{U}_{\bar{x}} \\
= & H\left(a_{1}, a_{2}\right)\left(\Psi_{y t}+\Psi_{y} \Psi_{y x}-\Psi_{x} \Psi_{y y}-\Psi_{y y y}-U_{t}-U U_{x}\right),
\end{aligned}
$$


for some function $H\left(a_{1}, a_{2}\right)$ which may be a constant. Substitution from Eqs. (9) and (11) into the left-side of (12) and rearrangement yields

$$
\begin{aligned}
& \left(C^{\Psi} / C^{y} C^{t}\right) \Psi_{y t}+\left[\left(C^{\Psi}\right)^{2} /\left(C^{y}\right)^{2} C^{x}\right]\left(\Psi_{y} \Psi_{y x}-\Psi_{x} \Psi_{y y}\right) \\
& -\left[C^{\Psi} /\left(C^{y}\right)^{3}\right] \Psi_{y y y}-\left(C^{U} / C^{t}\right) U_{t}-\left(C^{U} U\right)\left(C^{U} / C^{x}\right) U_{x} \\
= & H_{1}\left(a_{1}, a_{2}\right)\left(\Psi_{y t}+\Psi_{y} \Psi_{y x}-\Psi_{x} \Psi_{y y}-\Psi_{y y y}-U_{t}-U U_{x}\right)+R_{1}\left(a_{1}, a_{2}\right),
\end{aligned}
$$

where

$$
R_{1}\left(a_{1}, a_{2}\right)=K^{U}\left(C^{U} / C^{x}\right) U_{x}
$$

Thus, it follows that (13) is transformed invariantly whenever

$$
\begin{aligned}
\left(C^{\psi} / C^{y} C^{t}\right)=\left[\left(C^{\psi}\right)^{2} /\left(C^{y}\right)^{2} C^{x}\right] & =\left[C^{\psi} /\left(C^{y}\right)^{3}\right]=\left(C^{U} / C^{t}\right) \\
& =\left[\left(C^{U}\right)^{2} / C^{x}\right]=H_{1}\left(a_{1}, a_{2}\right) .
\end{aligned}
$$

The invariance of (13) implies $R_{1}=0$, which reduces to

$$
K^{U}=0
$$

In like manner the invariant transformation of (7) under (9) and (11) whenever there is a function $H_{2}\left(a_{1}, a_{2}\right)$ such that:

$$
\begin{aligned}
C^{T_{1}} & T_{1}\left(C^{\theta} / C^{t}\right) \theta_{t}+C^{\theta} \theta\left(C^{T_{1}} / C^{t}\right)\left(T_{1}\right)_{t}+C^{T_{1}} T_{1}\left(C^{\psi} / C^{y}\right)\left(C^{\theta} / C^{x}\right) \psi_{y} \theta_{x} \\
& +C^{\theta} \theta\left(C^{\psi} / C^{y}\right)\left(C^{T_{1}} / C^{x}\right) \psi_{y}\left(T_{1}\right)_{x}-C^{T_{1}} T_{1}\left(C^{\psi} / C^{x}\right)\left(C^{\theta} / C^{y}\right) \psi_{y} \theta_{y} \\
& -\frac{1}{P r} C^{T_{1}} T_{1}\left[C^{\theta} /\left(C^{y}\right)^{2}\right] \theta_{y y}=H_{2}\left(a_{1}, a_{2}\right)\left[T_{1} \theta_{t}+\theta\left(T_{1}\right)_{t}\right. \\
& \left.+T_{1} \psi_{y} \theta_{x}+\theta \psi_{y}\left(T_{1}\right)_{x}-T_{1} \psi_{x} \theta_{y}-\frac{1}{\operatorname{Pr}} T_{1} \theta_{y y}\right]+R_{2}\left(a_{1}, a_{2}\right)
\end{aligned}
$$

where

$$
\begin{aligned}
R_{2}\left(a_{1}, a_{2}\right)= & \left(K^{T_{1}} C^{\theta} / C^{t}\right) \theta_{t}+\left(K^{\theta} C^{T_{1}} / C^{t}\right)\left(T_{1}\right)_{t} \\
& +\left(K^{T_{1}} C^{\psi} C^{\theta} / C^{x} C^{y}\right) \psi_{y} \theta_{x}+\left(K^{\theta} C^{\psi} C^{T_{1}} / C^{x} C^{y}\right) \psi_{y}\left(T_{1}\right)_{x} \\
& -\left(K^{T_{1}} C^{\psi} C^{\theta} / C^{x} C^{y}\right) \psi_{x} \theta_{y}-\frac{1}{P r}\left(K^{T_{1}} C^{\theta} /\left(C^{y}\right)^{2}\right) \theta_{y y} .
\end{aligned}
$$

For invariability, we should have

$$
\left(C^{T_{1}} C^{\theta} / C^{t}\right)=\left(C^{T_{1}} C^{\psi} C^{\theta} / C^{x} C^{y}\right)=\left(C^{T_{1}} C^{\theta} /\left(C^{y}\right)^{2}\right)=H_{2}\left(a_{1}, a_{2}\right),
$$


implies $R_{2}=0$ and hence

$$
K^{T_{1}}=K^{\theta}=0
$$

Moreover, following Birkhoff (1960), the boundary conditions (8) is also invariant in form whenever the condition $K^{y}=0$ is appended to (15), (16), (19) and (20); that is

$$
\begin{aligned}
& \bar{y}=0: \quad \bar{\psi}_{\bar{y}}=0, \quad \bar{\psi}_{\bar{x}}=-\bar{v}_{\bar{w}}, \quad \bar{\theta}=1, \\
& \bar{y} \rightarrow \infty: \quad \bar{\psi}_{\bar{y}}=\bar{U}(\bar{x}, \bar{y}), \quad \bar{\theta}=0 .
\end{aligned}
$$

It is obvious that when $K^{y}=0$, the transformation of $\theta(x, 0, t)=1$ implies that $\bar{\theta}(\bar{x}, 0, \bar{t})=1$ which is only satisfied if

$$
C^{\theta}=1
$$

Combining Eqs. (15) and (19) and invoking the result (22), we get:

$$
C^{y}=1, C^{t}=1, \text { and } C^{\psi}=C^{x}=C^{U} .
$$

Thus, the foregoing restrictions indicate that groups which are of further interest are those in the class $C_{G}$, with the from:

$$
G:\left\{\begin{array}{l}
\bar{S}:\left\{\begin{array}{l}
\bar{x}=C^{x}\left(a_{1}, a_{2}\right) x+K^{x}\left(a_{1}, a_{2}\right) \\
\bar{y}=y \\
\bar{t}=t+K^{t}\left(a_{1}, a_{2}\right)
\end{array}\right. \\
\bar{\psi}=C^{x}\left(a_{1}, a_{2}\right) \psi \\
\bar{\theta}=\theta \\
\bar{T}_{1}=C^{T_{1}}\left(a_{1}, a_{2}\right) T_{1} \\
\bar{U}=C^{x}\left(a_{1}, a_{2}\right) U .
\end{array}\right.
$$

Thus, as may be directly verified, any two-parameter group with the form (24) transform (1) to (4) invariantly in the sense described.

\section{Complete Sets of Absolute Invariant}

The key feature of the systematic technique to be presented is the application of a basic theorem from group theory, Moran and Gaggioli (1968). To emphasize the essential features of the theorem in a relatively uncomplicated form, it is now 
quoted for the case of two-parameter groups $\bar{S}$, (24). At first, a function $\eta$ is an absolute invariant of a two-parameter group

$$
\begin{aligned}
\bar{S}:[\bar{x} & =C^{x}\left(a_{1}, a_{2}\right) x+K^{x}\left(a_{1}, a_{2}\right), \\
\bar{y} & =C^{y}\left(a_{1}, a_{2}\right) y+K^{y}\left(a_{1}, a_{2}\right), \\
\bar{t} & \left.=C^{t}\left(a_{1}, a_{2}\right) t+K^{t}\left(a_{1}, a_{2}\right)\right],
\end{aligned}
$$

if and only if $\eta$ satisfies the first order linear partial differential equations

$$
\begin{aligned}
& \left(\alpha_{1} x+\alpha_{2}\right) \frac{\partial \eta}{\partial x}+\left(\alpha_{3} y+\alpha_{4}\right) \frac{\partial \eta}{\partial y}+\left(\alpha_{5} t+\alpha_{6}\right) \frac{\partial \eta}{\partial t}=0 \\
& \left(\beta_{1} x+\beta_{2}\right) \frac{\partial \eta}{\partial x}+\left(\beta_{3} y+\beta_{4}\right) \frac{\partial \eta}{\partial y}+\left(\beta_{5} t+\beta_{6}\right) \frac{\partial \eta}{\partial t}=0
\end{aligned}
$$

where

$$
\begin{aligned}
\alpha_{1} & =\left[\frac{\partial C^{x}}{\partial a_{1}}\right]\left(a_{1}^{0}, a_{2}^{0}\right), & \beta_{1} & =\left[\frac{\partial C^{x}}{\partial a_{2}}\right]\left(a_{1}^{0}, a_{2}^{0}\right), \\
\alpha_{2} & =\left[\frac{\partial K^{x}}{\partial a_{1}}\right]\left(a_{1}^{0}, a_{2}^{0}\right), & \beta_{2} & =\left[\frac{\partial K^{x}}{\partial a_{2}}\right]\left(a_{1}^{0}, a_{2}^{0}\right), \ldots, \text { etc } .
\end{aligned}
$$

and wherein $\left(a_{1}^{0}, a_{2}^{0}\right)$ denote the values of $a_{1}$ and $a_{2}$ which yield the identity : $\bar{x}=x, \bar{y}=y$ and $\bar{t}=t$, see Moran and Gaggioli (1969) . By definition, for each of two-parameter groups $S$ in the class $C_{\dot{G}}$ there is one and only one functionally independent solution to (26), (the rank of the coefficient matrix for $\left[\frac{\partial \eta}{\partial x}, \frac{\partial \eta}{\partial y}, \frac{\partial \eta}{\partial t}\right]$ is two, the matrix has rank two whenever at least one of its two-by-two submatrices has a non-vanishing determinant). Furthermore, if $\eta \neq$ Const., is a solution to (26), for a group $S$, then every other solution to (26), for $S$, is given in the form $H(\eta)$ where $H$ is a differentiable function. It may be seen from (26) and the definitions of the constants $\alpha_{i}, \beta_{i}$ that differences between the group $S$ are reflected by the $\alpha^{\prime} s$ and $\beta^{\prime} s$ that is, in general, any particular group $S$ possesses a characteristic set of $\alpha^{\prime} s$ and $\beta^{\prime} s$; and consequently a characteristic absolute invariant $\eta$ is yielded by (26). Since $K^{y}=0$, then $\alpha_{4}=\beta_{4}=0$. Therefore, Eqs. (26) become

$$
\begin{aligned}
& \left(\alpha_{1} x+\alpha_{2}\right) \frac{\partial \eta}{\partial x}+\alpha_{3} y \frac{\partial \eta}{\partial y}+\left(\alpha_{5} t+\alpha_{6}\right) \frac{\partial \eta}{\partial t}=0 \\
& \left(\beta_{1} x+\beta_{2}\right) \frac{\partial \eta}{\partial x}+\beta_{3} y \frac{\partial \eta}{\partial y}+\left(\beta_{5} t+\beta_{6}\right) \frac{\partial \eta}{\partial t}=0 .
\end{aligned}
$$

\section{Derivation of Distinct Complete Sets}

The similarity analysis of (6-8) now proceeds for the particular case of twoparameter groups of the form (24). According to the basic theorem from group 
theory, Eq. (27) has one and only one solution, if at least one of the following conditions is satisfied

$$
\lambda_{31} x+\lambda_{32} \neq 0 \text { or } \lambda_{35} t+\lambda_{36} \neq 0 \text { or } \lambda_{15} x t+\lambda_{16} x+\lambda_{25} t+\lambda_{26} \neq 0,
$$

where

$$
\lambda_{i j}=\alpha_{i} \beta_{j}-\alpha_{j} \beta_{i}, \quad i, j=1,2, \ldots, 6 .
$$

For convenience, then, the system (27) will be rewritten in terms of the quantities given by (28); the result is:

$$
\begin{aligned}
& \left(\lambda_{31} x+\lambda_{32}\right) \frac{\partial \eta}{\partial x}+\left(\lambda_{35} t+\lambda_{36}\right) \frac{\partial \eta}{\partial t}=0, \\
& \left(\lambda_{31} x+\lambda_{32}\right) y \frac{\partial \eta}{\partial y}-\left(\lambda_{15} x t+\lambda_{16} x+\lambda_{25} t+\lambda_{26}\right) \frac{\partial \eta}{\partial t}=0 .
\end{aligned}
$$

According to conditions (28) the following cases arise.

\subsection{The first case}

From the transformations (24) and definitions of the $\alpha^{\prime} s, \beta^{\prime} s$ and $\lambda^{\prime} s$, we have the result

$$
\lambda_{31}=\lambda_{32}=\lambda_{35}=\lambda_{36}=\lambda_{15}=\lambda_{25}=0,
$$

which imply

$$
\lambda_{31} x+\lambda_{32}=0, \quad \lambda_{35} t+\lambda_{36}=0,
$$

then the condition (29) reduces to

$$
\lambda_{16} x+\lambda_{26} \neq 0
$$

Applying Eqs. (30-31) to Eqs. (29), we get

(i) the first equation of (29) is identically satisfied,

(ii) the second equation of (29) reduces to

$$
\frac{\partial \eta}{\partial t}=0
$$

For convenience, Eq. (27) can be rewritten in the form

$$
\left(\lambda_{16} x+\lambda_{26}\right) \frac{\partial \eta}{\partial x}=0
$$

and from (31), then Eq. (33) gives: 


$$
\frac{\partial \eta}{\partial x}=0
$$

From Eqs. (32) and (34) we have

$$
\eta=f(y) .
$$

Without loss of generality the independent absolute invariant in Eq. (35) may be assumed of the form:

$$
\eta=A y
$$

\subsection{The second case}

According to Eqs.(29), we study the following sub-cases:

6.2.1. Sub-case $\left(\lambda_{31} x+\lambda_{32}=0, \lambda_{35} t+\lambda_{36} \neq 0, \lambda_{15} x t+\lambda_{16} x+\lambda_{25} t+\lambda_{26} \neq 0\right)$

From Eq. (29) yields the solutions corresponding to this case is $\partial \eta / \partial t=0$, i.e., from Eq. (27) this yields a solution $\eta=\eta(x, y)$ and

$$
\begin{aligned}
& \left(\alpha_{1} x+\alpha_{2}\right) \frac{\partial \eta}{\partial x}+\alpha_{3} y \frac{\partial \eta}{\partial y}=0 \\
& \left(\beta_{1} x+\beta_{2}\right) \frac{\partial \eta}{\partial x}+\beta_{3} y \frac{\partial \eta}{\partial y}=0
\end{aligned}
$$

then

$$
\eta=y(A x+B)^{r}, r=-\frac{\alpha_{3}}{\alpha_{1}}=-\frac{\beta_{3}}{\beta_{1}}, A=\alpha_{1}=\beta_{1}, B=\alpha_{3}=\beta_{3} .
$$

6.2.2. Sub-case $\left(\lambda_{31} x+\lambda_{32} \neq 0, \lambda_{35} t+\lambda_{36}=0, \lambda_{15} x t+\lambda_{16} x+\lambda_{25} t+\lambda_{26} \neq 0\right)$

From Eq. (29) this yields a solution $\eta=\eta(y, t)$ and

$$
\lambda_{32} y \frac{\partial \eta}{\partial y}-\left(\lambda_{25} t+\lambda_{26}\right) \frac{\partial \eta}{\partial t}=0
$$

or

$$
\begin{aligned}
& \alpha_{3} y \frac{\partial \eta}{\partial y}-\left(\alpha_{5} t+\alpha_{6}\right) \frac{\partial \eta}{\partial t}=0 \\
& \beta_{3} y \frac{\partial \eta}{\partial y}-\left(\beta_{5} t+\beta_{6}\right) \frac{\partial \eta}{\partial t}=0
\end{aligned}
$$

then

$$
\eta=y(B t+A)^{r}, \quad r=\frac{\alpha_{3}}{\alpha_{5}}=\frac{\beta_{3}}{\beta_{5}}, A=\alpha_{6}, B=\alpha_{5}
$$


6.2.3. Sub-case $\left(\lambda_{31} x+\lambda_{32} \neq 0, \lambda_{35} t+\lambda_{36} \neq 0, \lambda_{15} x t+\lambda_{16} x+\lambda_{25} t+\lambda_{26}=0\right)$

From Eq. (29), the solution $\eta$ is a function of $x$ and $y$ (independent of $y$ ). This solution is unacceptable regarding the boundary conditions.

\subsection{The third case}

In this case, we suppose that some of the coefficients in (29) vanish

6.3.1. Sub-case $\left(\lambda_{31}=\lambda_{35}=0, \lambda_{32} \neq 0, \lambda_{36} \neq 0\right)$

In this sub-case Eqs. (29) become:

$$
\begin{aligned}
& \lambda_{32} \frac{\partial \eta}{\partial x}+\lambda_{36} \frac{\partial \eta}{\partial t}=0, \\
& \lambda_{32} y \frac{\partial \eta}{\partial y}-\left(\lambda_{15} x t+\lambda_{16} x+\lambda_{25} t+\lambda_{26}\right) \frac{\partial \eta}{\partial t}=0 .
\end{aligned}
$$

According to a well-known standard technique for linear partial differential equations, the first equation of (37) has the general solution

$$
\eta=f(y, \xi(x, t))
$$

where $f$ is an arbitrary function and

$$
\xi(x, t)=\lambda_{36} x-\lambda_{32} t=\text { Const } .
$$

From (38) and (39), the second equation of (37) becomes:

$$
y \frac{\partial f}{\partial y}+\left(\lambda_{15} x t+\lambda_{16} x+\lambda_{25} t+\lambda_{26}\right) \frac{\partial f}{\partial \xi}=0 .
$$

From (39) and if $\lambda_{15}=0$, Eq. (40) is written in the form

$$
y \frac{\partial f}{\partial y}+\left[\left(\frac{\lambda_{16}}{\lambda_{36}}\right) \xi+\lambda_{26}\right] \frac{\partial f}{\partial \xi}=0 .
$$

The solutions of (41) are readily found in the form

$$
f=\phi(y H(\xi)),
$$

where $H(\xi)$ is given via the ordinary differential equation

$$
-\left[\left(\frac{\lambda_{16}}{\lambda_{36}}\right) \xi+\lambda_{26}\right] \frac{d \ln H}{d \xi}=1,
$$

obtained by substitution of (42) into (43). Now, two distinct solutions may be obtained from (43) as special cases.

6.3.1a. Sub-case $\left(\lambda_{16} \neq 0\right)$ 
In this special sub-case, we have

$$
H=\left[\left(\frac{\lambda_{16}}{\lambda_{36}}\right) \xi+\lambda_{26}\right]^{-\lambda_{36} / \lambda_{16}} .
$$

From (38), (42) and (44), the solution $\eta$ is written in the form

$$
\eta=\phi\left(y(A x+B t+C)^{m}\right),
$$

where $m=-\frac{\lambda_{36}}{\lambda_{16}}$ and $A, B$ and $C$ are constants stand for $\lambda_{16}, \lambda_{25}$ and $\lambda_{26}$ respectively. Without loss of generality, the function $\phi$ can be taken to be the identity function. Thus

$$
\eta=y(A x+B t+C)^{m} .
$$

6.3.1b. Sub-case $\left(\lambda_{16}=0\right)$

For this special sub-case equation (43) yields the solution

$$
H=\exp \left(-\xi / \lambda_{26}\right)
$$

From (38), (42) and (44), the solution is written in the form

$$
\eta=\phi(y \exp (m x) \exp (-r t))
$$

where

$$
m=-\frac{\lambda_{36}}{\lambda_{26}}, \quad r=-\frac{\lambda_{32}}{\lambda_{26}} .
$$

In a similar manner, we get:

$$
\eta=y \exp (m x) \exp (-r t) .
$$

6.3.2. Sub-case $\left(\lambda_{31} \neq 0, \lambda_{35} \neq 0\right)$

In like manner, the formula of dimensionless coordinate $\eta$ is as the following:

$$
\eta=y(x+A)^{m}(t+B)^{r},
$$

where

$$
A=\frac{\lambda_{32}}{\lambda_{31}}, \quad B=\frac{\lambda_{16}}{\lambda_{15}}, m=-\frac{\lambda_{35}}{\lambda_{15}} \text { and } r=\frac{\lambda_{31}}{\lambda_{15}} .
$$

6.3.3. Sub-case $\left(\lambda_{31}=0, \lambda_{35} \neq 0, \lambda_{32} \neq 0\right)$

For this sub-case, we have 


$$
\eta=y(t+B)^{r} \exp (m x)
$$

where

$$
B=\frac{\lambda_{36}}{\lambda_{35}}, \quad r=\frac{\lambda_{32}}{\lambda_{25}} \text { and } m=-\frac{\lambda_{35}}{\lambda_{25}}
$$

6.3.4. Sub-case $\left(\lambda_{31} \neq 0, \lambda_{35}=0, \lambda_{36} \neq 0\right)$

For this sub-case, we have

$$
\eta=y(x+A)^{m} \exp (-r t)
$$

where

$$
A=\frac{\lambda_{32}}{\lambda_{31}}, m=-\lambda_{36}, \text { and } r=-\lambda_{31} .
$$

For the absolute invariants corresponding to the dependent variables $\psi, T_{1}, U$ and $\theta$. From (22), $\theta$ is itself an absolute invariant. Thus

$$
\chi_{1}(x, y, t ; \theta)=\theta(\eta) .
$$

A function $\chi_{2}(x, y ; \psi)$ is absolute invariant of two-parameter group if it satisfies the two first-order linear differential equations:

$$
\begin{aligned}
& \left(\alpha_{1} x+\alpha_{2}\right) \frac{\partial \chi_{2}}{\partial x}+\left(\alpha_{3} t+\alpha_{4}\right) \frac{\partial \chi_{2}}{\partial t}+\left(\alpha_{5} \psi+\alpha_{6}\right) \frac{\partial \chi_{2}}{\partial \psi}=0 \\
& \left(\beta_{1} x+\beta_{2}\right) \frac{\partial \chi_{2}}{\partial x}+\left(\beta_{3} t+\beta_{4}\right) \frac{\partial \chi_{2}}{\partial t}+\left(\beta_{5} \psi+\beta_{6}\right) \frac{\partial \chi_{2}}{\partial \psi}=0
\end{aligned}
$$

The solution of these equations gives

$$
\chi_{2}(x, t ; \psi)=\Phi_{1}\left(\psi / \Gamma_{1}(x, t)\right)=F(\eta) .
$$

In similar manner, we get

$$
\begin{aligned}
& \chi_{3}\left(x, t ; T_{1}\right)=\Phi_{2}\left(T_{1} / \Gamma_{2}(x, t)\right)=E(\eta), \\
& \chi_{4}(x, t ; U)=\Phi_{3}\left(U / \Gamma_{3}(x, t)\right)=H(\eta),
\end{aligned}
$$

where $\Gamma_{1}(x, t), \Gamma_{2}(x, t)$ and $\Gamma_{3}(x, t)$ are functions to be determined. Without loss of generality, the $\Phi^{\prime} s$ in (46) to (48) are selected to be the identity functions. Then we can express the functions $\psi(x, y, t), T_{1}(x, t)$ and $U(x, t)$ in terms of the absolute invariants $F(\eta), E(\eta)$ and $H(\eta)$ in the form.

(49) $\psi(x, y, t)=\Gamma_{1}(x, t) F(\eta), T_{1}(x, t)=\Gamma_{2}(x, t) E(\eta), U(x, t)=\Gamma_{3}(x, t) H(\eta)$. 
Since $\Gamma_{2}(x, t), \Gamma_{3}(x, t), T_{1}(x, t)$ and $U(x, t)$ are independent of $y$, whereas $\eta$ depends on $y$, it follows that $E$ and $H$ in (49) must be equal to a constant. Then

$$
\psi(x, y, t)=\Gamma_{1}(x, t) F(\eta), T_{1}(x, t)=T_{0} \Gamma_{2}(x, t), U(x, t)=U_{0} \Gamma_{3}(x, t) .
$$

The forms of the functions $\Gamma_{1}, \Gamma_{2}$ and $\Gamma_{3}$ are those for which the governing equations (6-8) reduce to ordinary differential equations.

\section{The Reduction to Ordinary Differential Equations}

By substituting of (50) into Eqs. (6-8) and if $\eta=y \pi(x, t)$, we have the following

$$
\begin{gathered}
F^{\prime \prime \prime}+C_{1}\left(F F^{\prime \prime}-F^{\prime 2}\right)-C_{2} F^{\prime 2}-C_{3}\left(\eta F^{\prime \prime}+F^{\prime}\right)-C_{4} F^{\prime}+C_{5}+C_{6}=0, \\
\frac{1}{P r} \theta^{\prime \prime}+C_{1} F \theta^{\prime}-C_{3} \eta \theta^{\prime}-C_{7} F^{\prime} \theta-C_{8} \theta=0
\end{gathered}
$$

with the boundary conditions:

$$
\begin{aligned}
& \eta=0: F^{\prime}=0, F=-\gamma, \theta=1, \\
& \eta=\infty: F^{\prime}=C_{9}, \theta=0,
\end{aligned}
$$

where the primes refer to differentiation with respect to $\eta$ and

$$
\begin{aligned}
& C_{1}=\frac{1}{\pi} \frac{\partial \Gamma_{1}}{\partial x}, \quad C_{2}=\frac{\Gamma_{1}}{\pi^{2}} \frac{\partial \pi}{\partial x}, \quad C_{3}=\frac{1}{\pi^{3}} \frac{\partial \pi}{\partial t}, \quad C_{4}=\frac{1}{\pi^{2} \Gamma_{1}} \frac{\partial \Gamma_{1}}{\partial t}, \\
& C_{5}=\frac{U_{0}}{\pi^{3} \Gamma_{1}} \frac{\partial \Gamma_{3}}{\partial t}, \quad C_{6}=\frac{U_{0}^{2} \Gamma_{3}}{\pi^{3} \Gamma_{1}} \frac{\partial \Gamma_{3}}{\partial x}, C_{7}=\frac{\Gamma_{1}}{\pi \Gamma_{2}} \frac{\partial \Gamma_{2}}{\partial x}, C_{8}=\frac{1}{\pi^{2} \Gamma_{2}} \frac{\partial \Gamma_{2}}{\partial t}, \\
& C_{9}=\frac{U_{0} \Gamma_{3}}{\pi \Gamma_{1}}, \quad \gamma=\frac{v_{w}}{\pi C_{1}} .
\end{aligned}
$$

The Equations (51-53) to be ordinary differential equations, the $C^{\prime} s$ are constants to be determined for each individual case corresponding to each set of absolute invariants and $\gamma$ is an arbitrary constant; see Mulolani and Rahman (2002). It remains to utilize each of the $\eta^{\prime} s$ in turn with (54) to evaluate the appearing $C^{\prime} s$ in the ordinary differential equations (51-53) and consequently to evaluate the corresponding expressions of the functions $\Gamma_{1}, \Gamma_{2}$ and $\Gamma_{3}$.

7.1. Reductions to ordinary differential equations. $(\eta=y \pi, \pi=A=1)$

In this section, we give the following sub-cases of $\Gamma_{1}$ and $\Gamma_{2}$. 
7.1.1. Solution when $\Gamma_{1}=\Gamma_{1}(x), \Gamma_{2}=\Gamma_{2}(x)$

For this sub-case and Eqs. (54), we have

$$
\Gamma_{1}=C_{1} x+K_{1}, C_{2}=C_{3}=C_{4}=C_{8}=0,
$$

where $K_{1}$ is an integral constant. By considering $C_{9}$ may be taken to be unity, we get the following

$$
\begin{aligned}
& U_{0} \Gamma_{3}=\Gamma_{1}, \quad C_{5}=0, C_{6}=C_{1}, \\
& \Gamma_{2}=K_{2}\left(C_{1} x+K_{1}\right)^{m}, \quad m=\frac{C_{7}}{C_{1}},
\end{aligned}
$$

where $K_{2}$ is an integral constant. By substituting the above-obtained values of the constants into Eqs. (51-53), we get:

$$
\begin{gathered}
F^{\prime \prime \prime}+C_{1}\left(F F^{\prime \prime}-F^{\prime 2}\right)+C_{1}=0, \\
\frac{1}{P r} \theta^{\prime \prime}+C_{1} F \theta^{\prime}-C_{7} F^{\prime} \theta=0,
\end{gathered}
$$

with the boundary conditions

$$
\begin{aligned}
& \eta=0: F^{\prime}=0, F=-\gamma, \theta=1, \\
& \eta=\infty: F^{\prime}=1, \theta=0 .
\end{aligned}
$$

The forms of $\psi, T_{w}$ and $U$ are as the following:

$$
\psi=\left(C_{1} x+K_{1}\right) F(\eta), T_{w}=T_{\infty}+T_{0} K_{2}\left(C_{1} x+K_{1}\right)^{m}, U=C_{1} x+K_{1} .
$$

By considering $C_{7}=0$, then Eqs. (55-57) become

$$
\begin{gathered}
F^{\prime \prime \prime}+C_{1}\left(F F^{\prime \prime}-F^{2}\right)+C_{1}=0, \\
\frac{1}{P r} \theta^{\prime \prime}+C_{1} F \theta^{\prime}=0,
\end{gathered}
$$

with the boundary conditions given by (57) and $T_{w}=$ Const.

When $C_{1}=1$, Eqs.(59-60) are reduced to special case of heat transfer for the Falkner- Skan flows, see Falkner and Skan (1931) and Frank (1991).

In this sub-case, the velocity components $u$ and $v$ are the following:

$$
u=\left(C_{1} x+K_{1}\right) F^{\prime}(\eta), v=-C_{1} F .
$$

7.1.2. Solution when $\Gamma_{1}=\Gamma_{1}(x), \Gamma_{2}=\Gamma_{2}(t)$

For this sub-case and Eqs. (54), we have 


$$
\begin{aligned}
& \Gamma_{1}=C_{1} x+K_{1}, \quad C_{2}=C_{3}=C_{4}=C_{7}=0, \\
& U_{0} \Gamma_{3}=\Gamma_{1}, C_{5}=0, \quad C_{6}=C_{1}, \quad \Gamma_{2}=K_{2} \exp \left(C_{8} t\right) .
\end{aligned}
$$

By substituting the above-obtained values of the constants into Eqs. (51-53), we get:

$$
\begin{gathered}
F^{\prime \prime \prime}+C_{1}\left(F F^{\prime \prime}-F^{\prime 2}\right)+C_{1}=0, \\
\frac{1}{P r} \theta^{\prime \prime}+C_{1} F \theta^{\prime}-C_{8} \theta=0,
\end{gathered}
$$

with the boundary conditions (57). The forms of $\psi, T_{w}$ and $U$ are as the following:

$$
\psi=\left(C_{1} x+K_{1}\right) F(\eta), T_{w}=T_{\infty}+T_{0} K_{2} \exp \left(C_{8} t\right), U=C_{1} x+K_{1} .
$$

This results will be referred to as the Hiemenz (1911) stagnation-point solution.

\subsubsection{Solution when $\Gamma_{1}=\Gamma_{1}(t), \Gamma_{2}=\Gamma_{2}(t)$}

For this sub-case, we have the following

$$
\begin{aligned}
& C_{1}=C_{2}=C_{3}=0, \Gamma_{1}=K_{1} \exp \left(C_{4} t\right), C_{4}=C_{5}, \\
& U_{0} \Gamma_{3}=\Gamma_{1}, C_{6}=C_{7}=0, \Gamma_{2}=K_{2} \exp \left(C_{8} t\right) .
\end{aligned}
$$

By substituting the above-obtained values of the constants into Eqs. (51-53), we get:

$$
\begin{gathered}
F^{\prime \prime \prime}-C_{4}\left(F^{\prime}-1\right)=0, \\
\frac{1}{P r} \theta^{\prime \prime}-C_{8} \theta=0,
\end{gathered}
$$

with the boundary conditions, let $v_{w}=0$,

$$
\begin{aligned}
& \eta=0: F^{\prime}=0, F=0, \theta=1, \\
& \eta=\infty: F^{\prime}=1, \theta=0 .
\end{aligned}
$$

The forms of $\psi, T_{w}$ and $U$ are as the following:

$$
\psi=K_{1} \exp \left(C_{4} t\right) F(\eta), T_{w}=T_{\infty}+T_{0} K_{2} \exp \left(C_{8} t\right), U=K_{1} \exp \left(C_{4} t\right),
$$

where $K_{1}$ and $K_{2}$ are integral constants.

This sub-case referred to an exact analytic solution 


$$
\begin{aligned}
& u=K_{1} \exp \left(C_{4} t\right)\left(1-\exp \left(-\sqrt{C_{4}} y\right), v=0,\right. \\
& T=T_{\infty}+T_{0} K_{2} \exp \left(C_{8} t\right) \exp \left(-\sqrt{\operatorname{Pr} C_{8}} y\right) .
\end{aligned}
$$

It is also satisfies the full Navier-Stokes equation, see Ma and Hui (1990).

$$
\text { 7.1.4. Solution when } \Gamma_{1}=\Gamma_{1}(t), \Gamma_{2}=\Gamma_{2}(x)
$$

For this sub-case, we have the following

$$
\begin{aligned}
& C_{1}=C_{2}=C_{3}=0, \Gamma_{1}=K_{1} \exp \left(C_{4} t\right), C_{4}=C_{5}, \\
& U_{0} \Gamma_{3}=\Gamma_{1}, C_{6}=C_{8}=0, \Gamma_{2}=K_{2} \exp \left(C_{7} x / K_{1} \exp \left(C_{4} t\right)\right) .
\end{aligned}
$$

By substituting the above-obtained values of the constants into Eqs. (51-53), we get:

$$
\begin{aligned}
& F^{\prime \prime \prime}-C_{4}\left(F^{\prime}-1\right)=0, \\
& \frac{1}{P r} \theta^{\prime \prime}-C_{7} F^{\prime} \theta=0 .
\end{aligned}
$$

The forms of $\psi, T_{w}$ and $U$ are as the following:

$\psi=K_{1} \exp \left(C_{4} t\right) F(\eta), T_{w}=T_{\infty}+T_{0} K_{2} \exp \left(C_{7} x / K_{1} \exp \left(C_{4} t\right)\right), U=K_{1} \exp \left(C_{4} t\right)$.

\subsection{Reductions to ordinary differential equations. $(\eta=y \pi, \pi=\pi(x, t))$}

\subsubsection{Solution when $\pi=(A x+B)^{r}$}

The conditions (54) are satisfied only in the case of $\Gamma_{1}=\Gamma_{1}(x)$ and $\Gamma_{2}=\Gamma_{2}(x)$. For this case, we have the following

$$
\begin{aligned}
& C_{1}=K A(r+1), C_{2}=K A r, C_{6}=K A(2 r+1), \quad C_{9}=1, \\
& C_{3}=C_{4}=C_{5}=C_{8}=0,
\end{aligned}
$$

where $K$ is a constant. By substituting the above-obtained values of the constants into Eqs. (51-53), we get:

$$
\begin{gathered}
F^{\prime \prime \prime}+C_{1}\left[F F^{\prime \prime}-\frac{(2 r+1)}{(r+1)}\left(F^{2}-1\right)\right]=0, \\
\frac{1}{P r} \theta^{\prime \prime}+C_{1} F \theta^{\prime}-C_{7} F^{\prime} \theta=0,
\end{gathered}
$$

with the boundary conditions

$$
\begin{aligned}
& \eta=0: F^{\prime}=0, F=-\gamma, \theta=1, \\
& \eta=\infty: F^{\prime}=1, \theta=0 .
\end{aligned}
$$


The forms of $\psi, T_{w}$ and $U$ are as the following:

$$
\begin{aligned}
& \psi=K(A x+B)^{(r+1)} F(\eta), T_{w}=T_{\infty}+T_{0} K_{1}(A x+B)^{C_{7} / A K}, \\
& U=K(A x+B)^{(2 r+1)}
\end{aligned}
$$

where $K_{1}$ is an integral constant.

When $C_{1}=1, B=0, r=(n-1) / 2$ and $\Lambda=K A^{n}$, the present problem reduces to the general Falkner- Skan equation

$$
\begin{aligned}
& F^{\prime \prime \prime}+F F^{\prime \prime}+B_{r}\left(1-F^{\prime 2}\right)=0, \\
& \frac{1}{P r} \theta^{\prime \prime}+F \theta^{\prime}-C_{7} F^{\prime} \theta=0,
\end{aligned}
$$

with $B_{r}=\frac{2 n}{n+1}, \gamma=0$ and the boundary conditions

$$
\begin{aligned}
& \eta=0: F^{\prime}=0, F=0, \quad \theta=1, \\
& \eta=\infty: F^{\prime}=1, \theta=0 .
\end{aligned}
$$

The forms of $\psi, T_{w}$ and $U$ are as the following:

$$
\psi=K(A x)^{(n+1) / 2} F(\eta), T_{w}=T_{\infty}+T_{0} K_{1}(A x)^{C_{7} / A K}, U=\Lambda x^{n} .
$$

The Falkner-Skan equation as well as the famous Blasius (1908) solution when $n=0$ and the Hiemenz (1911) stagnation point flow when $n=1$. The latter is also an exact solution to the full Navier-Stokes equations, see Ma and Hui (1990) and Schlichting and Gersten (2000).

\subsubsection{Solution when $\pi=(B t+A)^{r}$}

The conditions (54) are satisfied only in the case of $\Gamma_{1}=\Gamma_{1}(x, t), \Gamma_{2}=\Gamma_{2}(x, t)$ and $r=-\frac{1}{2}$. For this sub-case, we have the following

$$
\begin{aligned}
& C_{2}=0, \quad C_{6}=C_{1}, \quad C_{9}=1, \\
& C_{3}=C_{4}=\frac{C_{5}}{2}=-\frac{B}{2} \text { and } C_{8}=0 .
\end{aligned}
$$

By substituting the above-obtained values of the constants into Eqs.(51-53), we get:

$$
\begin{gathered}
F^{\prime \prime \prime}+C_{1}\left(F F^{\prime \prime}-F^{\prime 2}+1\right)+\frac{B}{2}\left(\eta F^{\prime \prime}+2 F^{\prime}-2\right)=0, \\
\frac{1}{P r} \theta^{\prime \prime}+C_{1} F \theta^{\prime}-C_{7} F^{\prime} \theta+\frac{B}{2} \eta \theta^{\prime}=0,
\end{gathered}
$$

with the boundary conditions: 


$$
\begin{aligned}
& \eta=0: F^{\prime}=0, F=-\gamma, \theta=1, \\
& \eta=\infty: F^{\prime}=1, \theta=0 .
\end{aligned}
$$

The forms of $\psi, T_{w}$ and $U$ are as the following:

$\psi=C_{1} \frac{\left(x+K_{1}\right)}{\sqrt{B t+A}} F(\eta), T_{w}=T_{\infty}+T_{0} K_{2}\left(x+K_{1}\right)^{C_{7} / C_{1}}, U=C_{1} \frac{\left(x+K_{1}\right)}{(B t+A)}$,

where $K_{1}$ and $K_{2}$ are integral constants.

When $C_{1}=1$ and $C_{7}=0$, the present problem reduces to problem of nonsteady plane stagnation point flow with hard blowing, see Rajappa (1979), where $B$ is called the acceleration parameter. The forms of $\psi, T_{w}$ and $U$ are as the following:

$$
\psi=\frac{\left(x+K_{1}\right)}{\sqrt{B t+A}} F(\eta), T_{w}=\text { Const. }, \quad U=\frac{\left(x+K_{1}\right)}{(B t+A)} .
$$

For the above sub-case, the boundary-layer characteristics are, the velocity components $u$ and $v$ are the following:

$$
u=\frac{\left(x+K_{1}\right)}{(B t+A)} F^{\prime}(\eta), \quad v=-\frac{x}{\sqrt{B t+A}} F(\eta) .
$$

When $B=1$ and $\gamma=0$, the present problem (70-72) referred to as the unsteady separated stagnation point flow, see Ma and Hui (1990).

\subsubsection{Solution when $\pi=(A x+B t+C)^{m}$}

The conditions (54) are satisfied only in the case of $\Gamma_{1}=\Gamma_{1}(x, t), \Gamma_{2}=\Gamma_{2}(x, t)$ and $m=-\frac{1}{2}$. For this sub-case, we have the following

$$
\begin{aligned}
& C_{1}=\frac{K A}{2}, \quad C_{2}=-\frac{K A}{2}, \quad C_{3}=-\frac{B}{2}, \quad C_{4}=\frac{B}{2}, \\
& C_{5}=0, \quad C_{6}=0, \quad C_{8}=\frac{B C_{7}}{A K}, \quad C_{9}=1,
\end{aligned}
$$

where $K$ is a constant. By substituting the above-obtained values of the constants into Eqs. (51-53), we get:

$$
\begin{gathered}
F^{\prime \prime \prime}+\frac{K A}{2} F F^{\prime \prime}+\frac{B}{2} \eta F^{\prime \prime}=0 \\
\frac{1}{P r} \theta^{\prime \prime}+\left(\frac{K A}{2} F+\frac{B}{2} \eta\right) \theta^{\prime}-C_{7}\left(F^{\prime}+\frac{B}{A K}\right) \theta=0,
\end{gathered}
$$

with the boundary conditions

$$
\begin{aligned}
& \eta=0: F^{\prime}=0, F=-\gamma, \theta=1, \\
& \eta=\infty: F^{\prime}=1, \theta=0 .
\end{aligned}
$$


The forms of $\psi, T_{w}$ and $U$ are as the following:

$$
\begin{aligned}
& \psi=K \sqrt{(A x+B t+C)} F(\eta), \\
& T_{w}=T_{\infty}+T_{0} K_{1}(A x+B t+C)^{C_{7} / A K}, \quad U=\text { Const. }
\end{aligned}
$$

For steady case $\left(B=0, C_{7}=0\right)$, we assume that $K=1, A=1$, the ordinary differential equations (73-76) retrieve Blasius's equation, see Blasius (1908).

$$
\begin{aligned}
& F^{\prime \prime \prime}+\frac{1}{2} F F^{\prime \prime}=0, \\
& \frac{1}{P r} \theta^{\prime \prime}+\frac{1}{2} F \theta^{\prime}=0,
\end{aligned}
$$

with the boundary conditions

$$
\begin{aligned}
& \eta=0: F^{\prime}=0, F=-\gamma, \theta=1, \\
& \eta=\infty: F^{\prime}=1, \theta=0 .
\end{aligned}
$$

The forms of $\psi, T_{w}$ and $U$ are as the following:

$$
\psi=\sqrt{x+C} F(\eta), T_{w}=\text { Const., } \quad U=\text { Const. }
$$

7.2.4. Solution when $\pi=\exp (m x) \exp (-r t)$

The conditions (54) are satisfied only in the case of $\Gamma_{1}=\Gamma_{1}(x), \Gamma_{2}=\Gamma_{2}(x)$ and $r=0$. For this sub-case, we have the following

$$
\begin{aligned}
& C_{1}=K m, \quad C_{2}=K m, \quad C_{3}=C_{4}=C_{5}=0, \\
& C_{6}=2 K m, \quad C_{8}=\frac{B C_{7}}{A K}, \quad C_{9}=1,
\end{aligned}
$$

where $K$ is a constant. For this case, Eqs. (51-53) will take the following form:

$$
\begin{aligned}
& F^{\prime \prime \prime}+K m\left(F F^{\prime \prime}-2 F^{\prime 2}+2\right)=0, \\
& \frac{1}{P r} \theta^{\prime \prime}+K m F \theta^{\prime}-C_{7} F^{\prime} \theta=0,
\end{aligned}
$$

with the boundary conditions:

$$
\begin{aligned}
& \eta=0: F^{\prime}=0, F=-\gamma, \theta=1, \\
& \eta=\infty: F^{\prime}=1, \theta=0,
\end{aligned}
$$

and the forms of $\psi, T_{w}$ and $U$ are as the following:

$\psi=K \exp (m x) F(\eta), T_{w}=T_{\infty}+T_{0} K_{1} \exp \left(C_{7} x / K\right), \quad U=K \exp (2 m x)$. 
When $m=1, K=1$ and $C_{1}=1$, this well-known reduction of the steady boundary-layer equations, which has an associated external velocity field given $U=$ $\exp (2 x)$, is a limiting case of the Falkner-Skan solutions.

\subsubsection{Solution when $\pi=(x+A)^{m}(t+B)^{r}$}

The conditions (54) are satisfied only in the case of $\Gamma_{1}=\Gamma_{1}(x, t), \Gamma_{2}=\Gamma_{2}(x, t)$ and $r=-\frac{1}{2}, m=0$ or case of $\Gamma_{1}=\Gamma_{1}(x), \Gamma_{2}=\Gamma_{2}(x)$ and $r=0$.

\subsubsection{Solution when $\pi=(t+B)^{r} \exp (m x)$}

The conditions (54) are satisfied only in the case of $\Gamma_{1}=\Gamma_{1}(x, t), \Gamma_{2}=\Gamma_{2}(x, t)$ $r=-\frac{1}{2}$ and $m=0$ or case of $\Gamma_{1}=\Gamma_{1}(x), \Gamma_{2}=\Gamma_{2}(x)$ and $r=0$.

\subsubsection{Solution when $\pi=(x+A)^{m} \exp (-r t)$}

The conditions (54) are satisfied only in the case of $\Gamma_{1}=\Gamma_{1}(x), \Gamma_{2}=\Gamma_{2}(x)$ and $r=0$.

\section{Discussions}

In this work we have used the full form of the two-parameter group to present a comprehensive account of the forms of similarity reductions that can arise from unsteady laminar boundary-layer flow near a stagnation point. Solutions are obtained for the main stream patterns and wall temperature variation for boundary layer flow with or without mass transfer from the wall.

The solutions are considered complete for the sake of this investigation when the steady and unsteady boundary layer equations have been transformed into ordinary differential equations. The ordinary differential equations so derived are the aim of this work.

There are many configurations of the main stream flow and variation of wall temperature for which the similarity variable $\eta,(\eta=y \pi)$ here could be obtained. The solution with $\pi=A=1$, (Sub-sections 7.1.1 and 7.1.2) represents the similarity solution for the steady-state flows which agreement with the results given by Birkhoff (1960). Further, the solution with $\pi=(A x+B)^{r}$ represents the similarity solution for the steady flow and heat transfer in the boundary layer near a stagnation point (see Sub-section 7.2.1). As a special case when $C_{1}=r=1$ and $C_{7}=1$, the equations (67-69) reduce to Hiemenz flow (see Schlichting and Gersten (2000)).

The similarity solutions for unsteady stagnation point flow are obtained when $\pi=(B t+A)^{r}$, with $r=-\frac{1}{2}, C_{1}=1$ and $C_{7}=0$ with hard blowing $F_{w}=$ $-v_{w} \sqrt{B t+A}$. The similarity variables of unsteady case under these conditions are agreement with Birkhoff (1960) and Rajappa (1979). Also, the uniform wall 
temperature ( $T_{w}=$ Const. $)$. When $B=1$ and $\gamma=0$, the results referred to as the unsteady separated stagnation point flow, see Ma and Hui (1990), and steady case $(B=0)$ are included as special cases.

A new forms for the similarity representations are also obtained for both steady and unsteady in which the similarity variables $\pi=(A x+B t+C)^{m}$. When $m=-\frac{1}{2}$ refers to nonsteady case with the wall temperature $T_{w}=T_{\infty}+T_{0} K_{1}(A x+B t+$ $C)^{C_{7} / A K}$, and the main stream is constant and when $\left(B=0, C_{7}=0\right)$, refer to the steady case, the system of equations reduce to the Blasius's equations with heat transfer (see Blasius (1908)).

For the solution when $\pi=\exp (m x) \exp (-r t)$. The similarity solution is obtained when $r=0$. This refers to steady state with the wall temperature $T_{w}=T_{\infty}+T_{0}$ $K_{1} \exp \left(C_{7} x_{/ K}\right.$ and the main stream is $U=K \exp (2 m x)$. This may also be regarded as the limiting case of $U=\Lambda x^{n}$ as $n \rightarrow \infty$, see Ma and Hui (1990).

To close we have used the invariance of Eqs. (6-8) under the two parameter group of transformations to reduces Eqs.(1-4) to an ordinary differential equation. We have shown that our solutions include all the previously known solutions as special cases. In addition many new solutions are found which are also solutions to the full Navier-Stokes equation. In works reported elsewhere we have undertaken examinations into the possible forms of similarity solutions of the unsteady incompressible Navier-Stokes equations. The possible forms of ordinary differential equation can not be solved analytically, a numerical solution can be obtained using a fourth-order Runge-Kutta scheme and the gradient method.

\section{ACKNOWLEDGMENT}

We express our gratitude to the (unknown) referee for his comments and suggestions.

\section{REFERENCES}

1. M. B. Abd-El-Malek and S. M. A. El-Mansi, Group theoretic methods applied to Burgers' equation, J. Comput. Appl. Math., 115 (2000), 1-12.

2. G. Birkhoff, Hydrodynamics, Princeton University Press, Princeton, New Jersey, 1960.

3. H. Blasius, Grenzschichten in Flussigkeiten mit kleiner Reibung, Z. Math. U. Phys., 56 (1908), 1-37.

4. G. I. Burde, A class of solutions of the boundary-layer equations, Fluid Dynamics, 25 (1990), 201-207.

5. G. I. Burde, The construction of special explicit solutions of the boundary-layer equations-steady flows, $Q$. Jl. Mech. Appl. Math., 47 (1994), 247-260. 
6. G. I. Burde, The construction of special explicit solutions of the boundary-layer equations- unsteady flows, Ibid., 48 (1995), 611-633.

7. G. I. Burde, New similarity reductions of the steady-state boundary-layer equations, J. Phys. A. Math. Gen., 29 (1996), 1665-1683.

8. B. J. Cantwell, Introduction to Symmetry Analysis, Cambridge University Press, Cambridge, 2003.

9. V. M. Falkner and S. W. Skan, Some approximate solutions of the boundary-layer equations, Philos. Mag., 12 (1931), 865-896.

10. M. W. Frank, Viscous Fluid Flow, Second ed., McGraw-Hill, New York, 1991.

11. C. W. Jones and E. J. Watson, In Laminar Boundary layer, Ed. L. Rosenhead, Clarendon Press, Oxford, 1963, Chapter V.

12. K. Hiemenz, Die Grenzschicht an einem in den gleichformigen Flussigkeitsstrom eingetauchten geraden Kreiszylinder. Dinglers Polytech. J., 326 (1911), 321-410.

13. P. K. H. Ma and W. H. Hui, Similarity solutions of the two-dimensional unsteady boundary-layer equations, J. Fluid Mech., 216 (1990), 537-559.

14. M.J. Moran and R.A. Gaggioli, A new systematic formalism for similarity analysis with application to boundary layer flows, U.S. Army Math. Research Center, Tech. Summary, Report No. 918, (1968).

15. M. J. Moran and R. A. Gaggioli, A new systematic formalism for similarity analysis, J. Eng. Math., 3 (1969), 151.

16. M. J. Moran and R. A. Gaggioli, Similarity for a real-gas flow via a new group formalism, Appl. Sci. Res., 22 (1970), 81-88.

17. I. Mulolani and M. Rahman, Similarity analysis for natural convection from a vertical plate with distributed wall concentration, I JMMS, 32 (2002), 319-334.

18. L. V. Ovsiannikov, Group Analysis of Differential Equations, Translation by Ed. W. F. Ames, Academic Press, New York, (1982).

19. N. R. Rajappa, Nonsteady plane stagnation point flow with hard blowing, ZAMM, 59 (1979), 471-473.

20. H. Schlichting and K. Gersten, Boundary Layer Theory; Translated by J. Kestin, Springer-Verlag, New York (2000).

I. A. Hassanien and A. A. Salama

Department of Mathematics,

Faculty of Science,

Assiut University,

Assiut 71516, Egypt

H. A. Hosham

Department of Mathematics,

Faculty of Science,

Al-Azhar University,

Assiut, Egypt 